\title{
The Emerging Role of Stereotactic Ablative Radiotherapy for Primary Renal Cell Carcinoma: A Systematic Review and Meta-Analysis
}

\author{
Rohann J.M. Correa ${ }^{a}$, Alexander V. Louie ${ }^{b}$, Nicholas G. Zaorsky ${ }^{c}$, Eric J. Lehrer ${ }^{d}$, Rodney Ellis ${ }^{e}$, \\ Lee Ponsky ${ }^{e}$, Irving Kaplan ${ }^{f}$, Anand Mahadevan ${ }^{g}$, William Chu ${ }^{b}$, Anand Swaminath ${ }^{n}$, \\ Raquibul Hannan ${ }^{h}$, Hiroshi Onishi ${ }^{i}$, Bin S. Teh ${ }^{j}$, Alexander Muacevic ${ }^{k}$, Simon S. Lo ${ }^{l}$, \\ Michael Staehler $^{k}$, Shankar Siva ${ }^{m, *}$
}

\begin{abstract}
${ }^{a}$ Department of Radiation Oncology, London Regional Cancer Program, London, Ontario, Canada; ${ }^{b}$ Department of Radiation Oncology, Sunnybrook Health Sciences Centre and the University of Toronto, Toronto, Ontario, Canada; ${ }^{\mathrm{c}}$ Department of Radiation Oncology, Penn State Cancer Institute, Hershey, PA, USA; ${ }^{\mathrm{d}}$ Department of Radiation Oncology, Icahn School of Medicine at Mount Sinai, New York, NY, USA; ${ }^{\mathrm{e}}$ University Hospitals Seidman Cancer Center, Case Comprehensive Cancer Center, Cleveland, OH, USA; ${ }^{\mathrm{f}}$ Beth Israel Deaconess Medical Center, Boston, MA, USA; ${ }^{\mathrm{g}}$ Geisinger Health, Danville, PA, USA; ${ }^{\mathrm{h}}$ Department of Radiation Oncology, Harold C. Simmons Cancer Center, University of Texas Southwestern Medical Center, Dallas, TX, USA; ${ }^{\mathrm{i}}$ Department of Radiology, University of Yamanashi, Yamanashi, Japan; ${ }^{\mathrm{j}}$ Department of Radiation Oncology, Houston Methodist Hospital, Cancer Center and Research Institute, Houston, TX, USA; ${ }^{\mathrm{k}}$ University of Munich Hospitals, Munich, Germany; ${ }^{1}$ Department of Radiation Oncology, University of Washington School of Medicine, Seattle, Washington, USA; ${ }^{\mathrm{m}}$ Division of Radiation Oncology \& Cancer Imaging, Peter MacCallum Cancer Centre, and University of Melbourne,

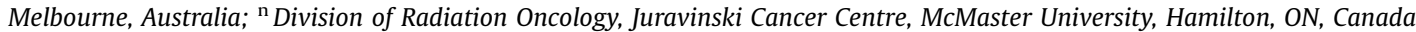

\section{Article info}

\section{Article history:}

Accepted June 5, 2019

\section{Associate Editor: Malte Rieken}

\section{Keywords:}

Stereotactic ablative

radiotherapy

Stereotactic body radiotherapy

Kidney cancer

Renal cell carcinoma

Radiosurgery

Small renal mass

\begin{abstract}
Context: Stereotactic ablative radiotherapy (SABR) is an emerging treatment option for primary renal cell carcinoma (RCC).

Objective: To systematically review the literature on SABR for primary RCC and perform a meta-analysis evaluating local control (LC), toxicity, and renal function.

Evidence acquisition: A PROSPERO-registered (\#115573), Preferred Reporting Items for Systematic Review and Meta-analyses (PRISMA)-based systematic review of the literature was conducted (1995-2019). Studies of SABR targeting primary RCC tumors were included, while those targeting only metastases were excluded. The primary outcome was LC defined as tumor size reduction and/or absence of local progression. Secondary outcomes included toxicity (Common Terminology Criteria for Adverse Events) and renal function (change in estimated glomerular filtration rate [eGFR]). Weighted random-effect meta-analyses using the DerSimonian and Laird method were conducted for primary and secondary outcomes. The $I^{2}$ statistic and Cochran's $Q$ test were used to assess heterogeneity.

Evidence synthesis: From 2386 PubMed entries and 924 meeting abstracts, 26 studies were identified (11 prospective trials), including 383 tumors in 372 patients, most of whom were deemed inoperable. Weighted averages (ranges) of median follow-up, median age, and mean tumor size were 28.0 (5.8-79.2) mo, $70.4(62-83) \mathrm{yr}$, and 4.6 $(2.3-9.5) \mathrm{cm}$, respectively. RCC histology was confirmed in $78.9 \%$ of patients who

* Corresponding author. Division of Radiation Oncology \& Cancer Imaging, Peter MacCallum Cancer Centre, 305 Grattan Street, Melbourne, Melbourne, Australia. Tel.: +61 3 8559 5000; Fax: +61 96561424. E-mail address: Shankar.Siva@petermac.org (S. Siva).
\end{abstract}


underwent pretreatment biopsy. Dose fractionation varied, but $26 \mathrm{~Gy}$ in one fraction and $40 \mathrm{~Gy}$ in five fractions were most common. The random-effect estimates for LC, grade $3-4$ toxicity, and post-SABR eGFR change were $97.2 \%$ ( $95 \%$ confidence interval $[\mathrm{CI}]$ : $\left.93.9-99.5 \%, I^{2}=20 \%\right), 1.5 \%\left(95 \% \mathrm{CI}: 0-4.3 \%, I^{2}=0 \%\right)$, and $-7.7 \mathrm{ml} / \mathrm{min}(95 \% \mathrm{CI}:-12.5$ to $-2.8, I^{2}=2 \%$, respectively, and heterogeneity was minimal. Six patients with preexisting renal dysfunction (2.9\%) required dialysis.

Conclusions: Renal SABR is locally effective and associated with low toxicity rates for primary RCC, despite treatment of larger tumors in older, mostly medically inoperable patients.

Patient summary: Stereotactic ablative radiotherapy is a high-precision, noninvasive radiation treatment requiring few outpatient visits, and represents a safe and effective management option for primary renal cell carcinoma.

(C) 2019 European Association of Urology. Published by Elsevier B.V. All rights reserved.

\section{Introduction}

\subsection{Renal cell carcinoma: rising incidence in the elderly}

Renal cell carcinoma (RCC) continues to increase in incidence, a trend partly attributed to greater utilization of noninvasive imaging incidentally identifying small renal masses [1]. The greatest increase in incidence is now seen in patients aged over $70 \mathrm{yr}$; in the UK from 2013 to 2155, 36\% of new cases occurred in people aged $\geq 75 \mathrm{yr}$ [1]. Increased age is also associated with a greater burden of renal function-relevant comorbidities (eg, hypertension, diabetes, and chronic kidney disease [CKD]) and cardiovascular disease that may preclude major surgery and associated anesthesia.

Although the recommended standard of care for localized RCC is surgical extirpation via partial or radical nephrectomy (PN or RN), elderly and frail patients with medical comorbidities may be unsuitable for surgery. Nonetheless, the highest cancer-specific RCC mortality rates are seen in older patients [1]. Moreover, elderly patients with stage I disease are at a higher risk of cancer-specific mortality (up to 3.8-fold), suggesting that even early-stage RCC can behave aggressively in the elderly [2]. Therefore, such patients are in need of definitive management options, possibly after an initial period of active surveillance (AS).

\subsection{Nonsurgical management alternatives}

\subsubsection{Active Surveillance with delayed intervention}

AS is commonly utilized in elderly, frail patients with decreased performance status, higher burden of comorbidities, and small tumors. This strategy is considered safe for small renal masses $(<4 \mathrm{~cm})$, as there is a low risk $(1-2 \%)$ of developing metastatic disease and little benefit to surgery in patients aged $>75 \mathrm{yr}$ due to high competing-cause mortality [3]. However, after a period of monitoring, up to $42 \%$ of patients on AS may require delayed intervention, often triggered by tumor growth [4]. In this setting, a larger tumor size portends a poorer prognosis: a recent retrospective analysis of 2650 patients demonstrated that a larger tumor size increased the likelihood of aggressive histology, which was associated with a $15 \%$ decrease in cancer-specific survival [5]. A recent review of 69 publications of AS by Ristau et al [6] suggests that delayed intervention after a period of
AS should thus be considered for size $>3-4 \mathrm{~cm}$, rapid growth $(>0.5 \mathrm{~cm} / \mathrm{yr})$, or a malignant biopsy result.

\subsubsection{Thermal ablation}

Definitive management-either upfront or after a period of AS-is more likely to involve cryoablation (CA) or radiofrequency ablation (RFA) in elderly or medically comorbid patients. These techniques have important limitations, specifically with respect to tumor size and location. For larger (T1b) tumors, local control (LC) rates for both TA modalities are lower [7,8]. Furthermore, major complications such as hemorrhage (more common with CA) are more likely for tumors with a central/perihilar location or larger tumors [9]. Thus, while thermal ablation (TA) is an accepted option in this setting, some patients may still have suboptimal outcomes with this approach.

\subsection{Stereotactic ablative radiotherapy: an emerging noninvasive option}

A higher dose per fraction (ie, an "ablative" dose) can yield dramatic cytotoxic effects in RCC, which has traditionally been viewed as a radioresistant tumor. Ablative doses overcome the perceived radioresistance of RCC partly by invoking nontraditional mechanisms of tumor cytotoxicity, namely activation of antitumor host immunity and induction of tumor endothelial cell apoptosis [10]. These mechanisms are particularly relevant in RCC with its rich vascularity and high insertion/deletion mutational burden, rendering RCC particularly conducive to techniques that result in microvascular disruption and engagement of host antitumor immunity [10]. Correspondingly, in preclinical mouse studies using RCC cell lines, ablative doses yield dramatic tumor regression [11]. Despite these encouraging data, however, delivery of such high doses was initially precluded by adjacent normal tissues sensitive to radiation injury.

Stereotactic ablative radiotherapy (SABR), synonymous with stereotactic body radiotherapy, delivers extremely high doses of conformal radiation in a single fraction or a few fractions with a rapid dose fall-off. Technological advancements over the last $20 \mathrm{yr}$ in radiation treatment planning, image guidance, and tumor motion tracking now allow "sculpting" of radiation doses around a target, sparing the normal organs at risk (Fig. 1). From the patient's 
perspective, this is a noninvasive, outpatient procedure requiring a few short visits. These technological advancements were subsequently applied to primary RCC: a systematic review by Siva et al (2012) [12] of these early clinical studies revealed that SABR achieves excellent clinical efficacy with minimal toxicity. Since then, nine prospective trials have been published. Therefore, a meta-analysis of the current literature on SABR for primary RCC is warranted to obtain contemporary estimates of efficacy, toxicity, and renal function impact.

\section{Evidence acquisition}

\subsection{Search strategy}

We conducted a Preferred Reporting Items for Systematic Review and Meta-analyses (PRISMA)-based, PROSPEROregistered (\#115573) search of PubMed/MEDLINE databases. A broad search strategy was employed using both medical subject heading (MeSH) and "text word" approaches to capture recent entries not yet $\mathrm{MeSH}$ indexed. We included studies in the metastatic RCC (mRCC) setting, wherein SABR targeted the primary tumor. Secondary manual searches of meeting abstracts published in prominent radiation oncology journals were also conducted. Pertinent references from included articles or excluded reviews were also retrieved. The complete search strategy is listed in Supplementary Table 1 .
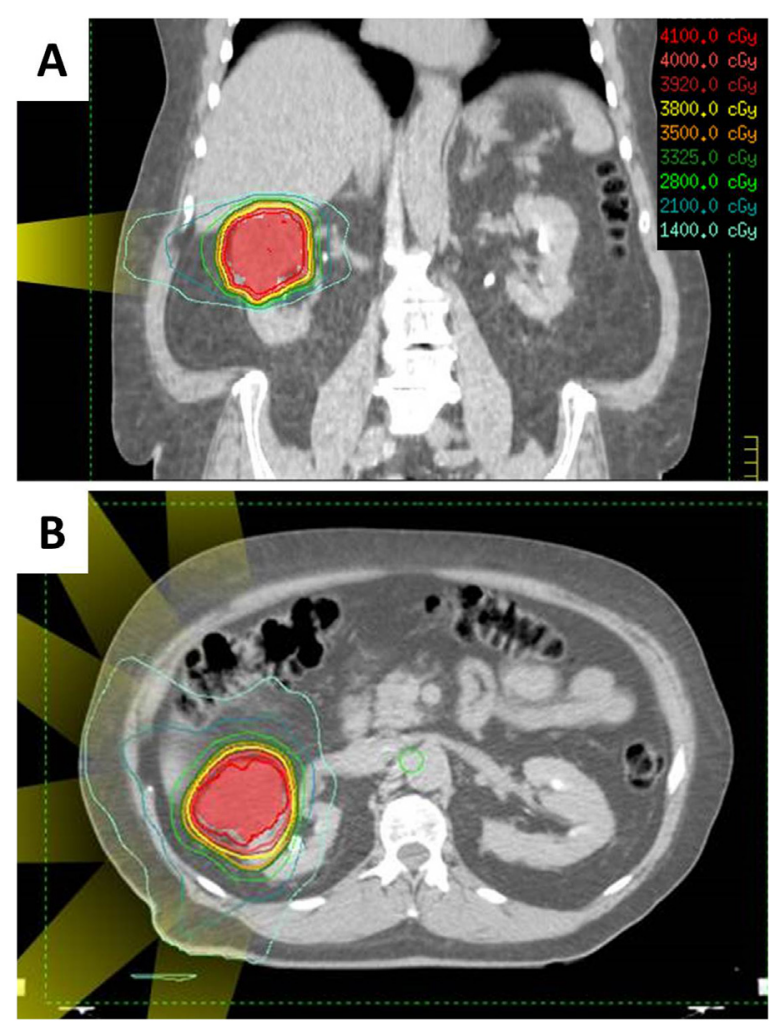

Fig. 1 - Stereotactic ablative radiotherapy for primary renal cell carcinoma (RCC). A representative radiotherapy plan treating a $6.5 \mathrm{~cm}$ right-sided RCC with $40 \mathrm{~Gy}$ in five fractions is shown in (A) axial and (B) coronal planes.

\subsection{Study eligibility}

We defined study eligibility using the patient population, intervention, comparator, outcome, and study design (PICOS) approach (Supplementary Table 2). Our search strategy and screening approach were complaint with PRISMA guidelines and involved two independent reviewers (R.J.M.C. and A.V.L.). We included published articles and abstracts that applied $\geq 5 \mathrm{~Gy}$ per fraction to the primary renal tumor and preferably obtained histological confirmation of RCC. Studies treating only extrarenal RCC metastases, reviews, meta-analyses, and case reports (less than two patients) were excluded. Dosimetric, motionmanagement, or radiotherapy (RT) planning studies were also excluded.

\subsection{Statistical methods}

The primary outcome was LC, defined as the proportion of stable disease or decreased tumor size (partial or complete response) at the last follow-up, as per the Response Evaluation Criteria in Solid Tumors (RECIST v1.1) criteria. If actuarial LC was also reported, the smaller of the two numbers (ie, worse LC) was used. Very little heterogeneity existed among studies with respect to the definition of LC, with all studies using radiographic, size-based evaluation criteria and $92 \%$ using a standardized system to define stable disease, and partial or complete response (eg, RECIST v1.1). Secondary outcomes included toxicity reported as Common Terminology Criteria for Adverse Events (CTCAE) and renal function reported as estimated glomerular filtration rate (eGFR).

Statistical analyses were performed using R Studio version 1.1.383 (R Studio, Boston, MA, USA). The Meta-Analysis for $\mathrm{R}$ (metafor) package version 2.0-0 was used to conduct the meta-analyses, tests for heterogeneity, and analysis of publication bias. The DerSimonian and Laird method was used to characterize the primary and secondary outcomes using weighted random-effect meta-analyses [13]. Proportions were calculated for LC and grade 3-4 toxicity. A random-effect model was chosen over a fixed-effect model as it is often preferred when the goal of the analysis is to aid in patient care decisions. Summary effect sizes for LC, grade 3-4 toxicity, and eGFR difference were depicted on forest diagrams with their associated 95\% confidence interval $(\mathrm{CI})$. Heterogeneity was assessed using both the $I^{2}$ statistic and Cochran $Q$ test. Heterogeneity was considered significant if $I^{2}>50 \%$ and the $p$ value of the $Q$ test was $<0.10$.

\section{Evidence synthesis}

Our search strategy yielded 2386 PubMed/MEDLINE articles and 924 meeting abstracts (Fig. 2). From these, 26 publications (22 plus four identified though screening of cited references) originating in eight different countries were included [14-39]. As listed in Table 1, publications (20 articles and six abstracts) included both retrospective series $(n=15)$ and prospective trials $(n=11)$. 


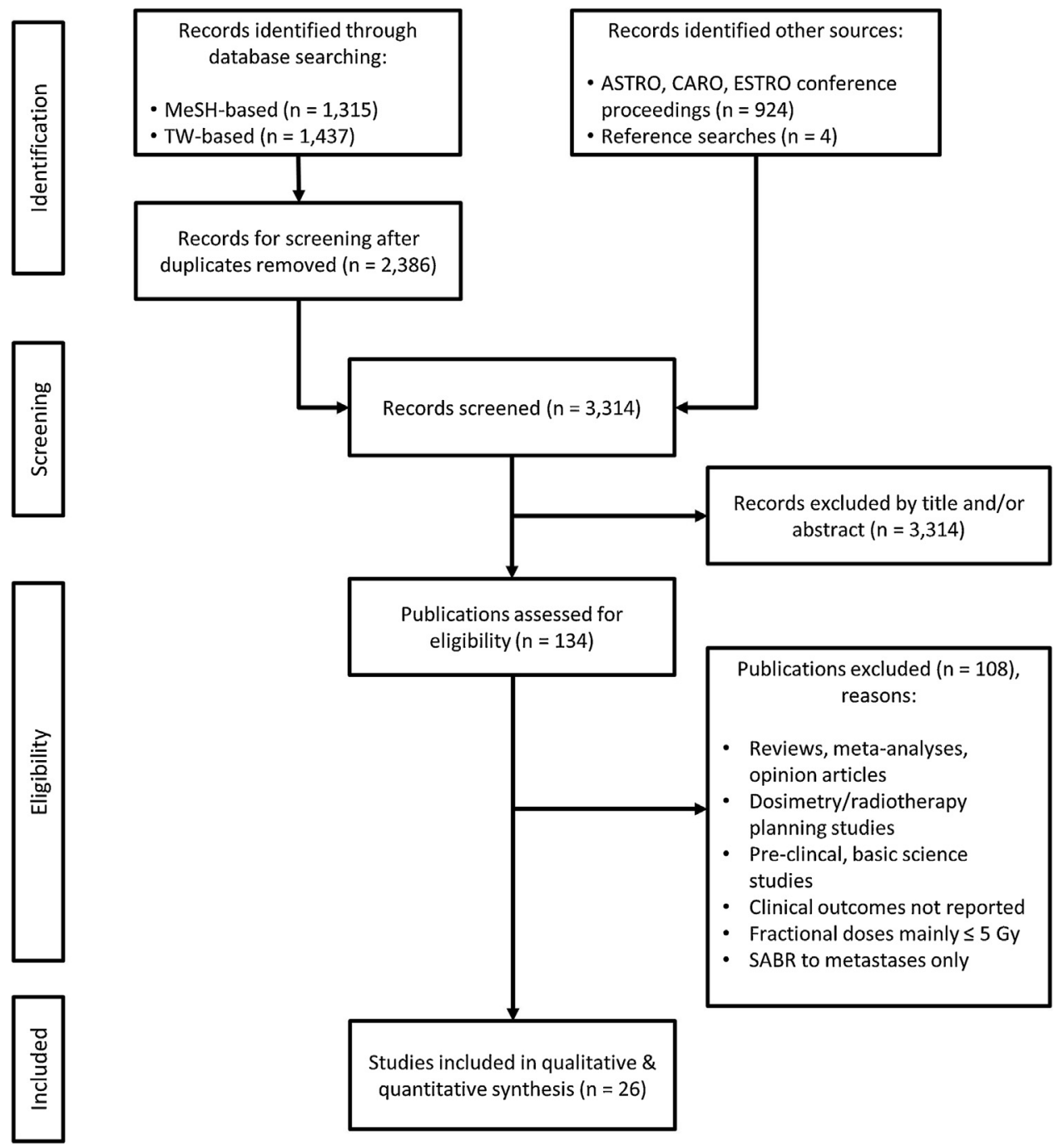

Fig. 2 - PRIMA flow diagram. MeSH = medical subject heading; PRISMA = Preferred Reporting Items for Systematic Review and Meta-analyses; $\mathrm{SABR}=$ stereotactic ablative radiotherapy; $T X=$ text word.

\subsection{Study and patient characteristics}

As summarized in Table 2, 26 eligible studies included 383 treated tumors among 372 patients. The majority ($n=300,80 \%$ ) had localized disease (stage I-II), but stage III-IV patients receiving SABR for a primary tumor were also included ( $n=72,20 \%$ ). Many stage I patients had been under AS but met criteria for delayed intervention. Most studies included pretreatment biopsy, which is the preferred standard of care. A biopsy confirmation rate of 78.9\% RCC histology was seen (in 191 of 242 patients where biopsy rates were reported). Of note, some patients in the study cohorts were elderly and medically inoperable, such that biopsy was stated to be not feasible. This biopsy confirmation rate is within a similar range to the TA literature: in a large meta-analysis, 494 of 600 (82.3\%) lesions managed with CA and 482 of 775 (62.2\%) lesions managed with RFA had preablation tissue confirmation [40]. If unbiopsied, serial enlargement on imaging was used to suggest a diagnosis of RCC. Radiological diagnosis was sometimes aided by additional imaging (kidney magnetic resonance imaging [MRI], angiography, and ultrasound) and reviewed by a second, independent radiologist [37]. Of note, 10 studies did not report biopsy confirmation rates; it is unclear whether these patients were histologically confirmed prior to treatment.

Weighted averages were calculated for key baseline characteristics. Weighted median follow-up was 28.0 (range: $5.8-$ 79.2 ) mo. Weighted median age was 70.3 (range: 62-83) yr, and most patients were deemed inoperable (21/23 studies, $91.3 \%)$ or declined surgery. The weighted mean tumor size was $4.6 \mathrm{~cm}$ (range: $2.3-9.5 \mathrm{~cm}, n=202$ ) in 15 studies where size (vs volume) was reported. A variety of dose-fractionation schedules were used across included studies: 30-40 Gy in three to five fractions and $26 \mathrm{~Gy}$ in one fraction were most common (Fig. 3).

\section{2. $\quad$ Local control}

The random-effect estimate for LC was 97.2\% (95\% CI: 93.9$99.5 \%$ ); there was a negligible amount of heterogeneity with 
Table 1 - Published reports of stereotactic ablative radiotherapy (SABR) for primary renal cell carcinoma.

\begin{tabular}{|c|c|c|c|c|c|c|c|c|c|c|c|c|c|c|c|}
\hline \multirow[t]{2}{*}{ 1st author (year) } & \multirow[t]{2}{*}{ Type } & \multirow{2}{*}{$\begin{array}{l}\text { Study } \\
\text { type }\end{array}$} & \multicolumn{3}{|c|}{ Patients } & \multirow{2}{*}{$\begin{array}{l}\text { Treated } \\
\text { tumors }\end{array}$} & \multirow{2}{*}{$\begin{array}{l}\text { Age } \\
\text { (yr) }\end{array}$} & \multirow{2}{*}{$\begin{array}{l}\text { Tumor size } \\
\left(\mathrm{cm} \text { or } \mathrm{cm}^{3}\right)\end{array}$} & \multirow{2}{*}{$\begin{array}{l}\text { Follow-Up } \\
\text { (mo) }\end{array}$} & \multirow{2}{*}{$\begin{array}{l}\text { Dose (Gy)/ } \\
\text { fractions }\end{array}$} & \multicolumn{2}{|c|}{ Toxicity (CTCAE) } & \multicolumn{2}{|c|}{ Renal function } & \multirow{2}{*}{$\begin{array}{l}\text { Local } \\
\text { control } \\
\text { (\%) }\end{array}$} \\
\hline & & & Total & $\begin{array}{l}\text { Stage } \\
\text { I-II }\end{array}$ & $\begin{array}{l}\text { Stage } \\
\text { III-IV }\end{array}$ & & & & & & Grade 3 & Grade 4 & $\begin{array}{l}\text { Pre-SABR } \\
\text { eGFR } \\
\text { (ml/min) }\end{array}$ & $\begin{array}{l}\text { Post-SABR } \\
\text { change in } \\
\text { eGFR (ml/min) }\end{array}$ & \\
\hline Qian (2003) [14] & $\mathrm{Ab}$ & $\mathrm{R}$ & 20 & $\mathrm{NR}$ & NR & 27 & 62 & $367 \mathrm{~cm}^{3}$ & 12 & $40 / 5$ & NR & NR & NR & NR & 9 \\
\hline Beitler (2004) [15] & $\mathrm{Ar}$ & $\mathrm{R}$ & 9 & 7 & 2 & 11 & NR & $4.55 \mathrm{~cm}$ & 26.7 & $40 / 5$ & $\begin{array}{l}1 \times \text { Gastric } \\
\text { ulcer }\end{array}$ & 0 & NR & NR & 89 \\
\hline Wersall (2005) [16] & $\mathrm{Ar}$ & $\mathrm{R}$ & 8 & 5 & 3 & 8 & NR & NR & 37 & $40 / 5$ & $45 \%^{\mathrm{a}}$ & 0 & NR & Unchanged & 87.5 \\
\hline Gilson (2006) [17] & $\mathrm{Ab}$ & $\mathrm{R}$ & 33 & NR & NR & 33 & 62 & $356 \mathrm{~cm}^{3}$ & 17 & $40 / 5$ & NR & NR & NR & NR & 94 \\
\hline Svedman (2006) [18] & $\mathrm{Ar}$ & $P$ & 5 & 4 & 1 & 5 & 64 & NR & 52 & $\begin{array}{l}45 / 3 \\
40 / 4 \\
32 / 4 \\
30 / 2\end{array}$ & 0 & 0 & NR & NR & 80 \\
\hline Teh (2007) [19] & $\mathrm{Ar}$ & $\mathrm{R}$ & 2 & 0 & 2 & 2 & NR & $10-200 \mathrm{~cm}^{3}$ & 9 & $24-40 / 3-6$ & 0 & 0 & NR & Unchanged & 100 \\
\hline Svedman (2008) [20] & $\mathrm{Ar}$ & $\mathrm{R}$ & 7 & 1 & 6 & 7 & NR & $5.5 \mathrm{~cm}$ & 49 & $\begin{array}{l}30 / 3 \\
40 / 4\end{array}$ & 0 & 0 & $\begin{array}{l}\text { Normal } \\
\text { pre-SABR }\end{array}$ & $\begin{array}{l}\text { Unchanged (5 pts) } \\
\text { Cr increase (2 pts) }\end{array}$ & 85.7 \\
\hline Wurzer (2012) [21] & $\mathrm{Ab}$ & $\mathrm{R}$ & 23 & 23 & 0 & 23 & NR & NR & 37 & $40 / 5$ & 0 & 0 & NR & NR & 87 \\
\hline Chebotareva (2013) [22] & $\mathrm{Ab}$ & $\mathrm{R}$ & 18 & 0 & 18 & 18 & NR & $5-180 \mathrm{~cm}^{3}$ & 15.5 & $30-52 / 3-4$ & 0 & 0 & NR & $\begin{array}{l}\text { Unchanged (17 pts) } \\
\text { Cr increase (1 pt) }\end{array}$ & 100 \\
\hline Nair (2013) [23] & $\mathrm{Ar}$ & $\mathrm{R}$ & 2 & 2 & 0 & 2 & NR & $21.3 \mathrm{~cm}^{3}$ & 13 & $39 / 3$ & 0 & 0 & 38 & +6 & 100 \\
\hline McBride (2013) [24] & $\mathrm{Ab}$ & $\mathrm{P}$ & 15 & 15 & 0 & 15 & 75 & $3.4 \mathrm{~cm}$ & 36.7 & $21-48 / 3$ & 0 & 0 & 55 & -18 & 80 \\
\hline Lo (2014) [25] & $\mathrm{Ar}$ & $\mathrm{R}$ & 3 & 3 & 0 & 3 & 83 & $5.0 \mathrm{~cm}$ & 13 & $40 / 5$ & 0 & 0 & 28.7 & -6.7 & 100 \\
\hline Hanzly (2014) [26] & $\mathrm{Ar}$ & $\mathrm{R}$ & 4 & 4 & 0 & 4 & 72.5 & $5.1 \mathrm{~cm}$ & 21.5 & $15 / 1$ & 0 & 0 & NR & Unchanged & 75 \\
\hline Ponsky (2015) [27] & $\mathrm{Ar}$ & $\mathrm{P}$ & 19 & 19 & 0 & 19 & 77.6 & $57.9 \mathrm{~cm}^{3}$ & 13.7 & $24-48 / 4$ & $2 \times \mathrm{CKD}$ & $\begin{array}{l}1 \times \text { Duodenal } \\
\text { ulcer }\end{array}$ & NR & NR & 100 \\
\hline Staehler (2015) [28] & $\mathrm{Ar}$ & $P$ & 29 & 29 & 0 & 30 & 65.6 & $33.7 \mathrm{~cm}^{3}$ & 28.1 & $26 / 1$ & 0 & 0 & 76.8 & -6.5 & 100 \\
\hline Chang (2016) [29] & $\mathrm{Ar}$ & $\mathrm{R}$ & 16 & 10 & 6 & 16 & 73 & $4.0 \mathrm{~cm}$ & 19 & $30-40 / 5$ & 0 & $2 \times \mathrm{CKD}$ & $55^{\mathrm{b}}$ & $-7.92^{\mathrm{b}}$ & 100 \\
\hline Correa (2018) [30] & $\mathrm{Ar}$ & $\mathrm{R}$ & 11 & 2 & 9 & 11 & 79 & $9.5 \mathrm{~cm}$ & 46.8 & $25-40 / 5$ & $1 \times$ Nausea & 0 & 48.6 & -1.5 & 70 \\
\hline Sun (2016) [31] & $\mathrm{Ar}$ & $\mathrm{R}$ & 32 & 32 & 0 & 32 & 74.5 & $3.9 \mathrm{~cm}$ & 18.7 & $21-48 / 3$ & NR & NR & NR & NR & 92.7 \\
\hline Kaidar-Person (2017) [32] & $\mathrm{Ar}$ & $\mathrm{R}$ & 6 & 6 & 0 & 6 & 68.5 & $5.0 \mathrm{~cm}$ & 29.5 & $39 / 3$ & 0 & 0 & NR & Unchanged & 100 \\
\hline Siva (2017) [33] & $\mathrm{Ar}$ & $\mathrm{P}$ & 33 & 33 & 0 & 34 & 78 & $4.8 \mathrm{~cm}$ & 24 & $26 / 1$ or $42 / 3$ & $1 \times \mathrm{NS}$ & 0 & 54.7 & -11 & 97 \\
\hline Singh (2017) [34] & $\mathrm{Ar}$ & $P$ & 14 & 0 & 14 & 14 & 63.9 & NR & 1 & $15 / 1$ & $1 \times$ Anemia & 0 & NR & NR & NR \\
\hline Correa (2018) [35] & $\mathrm{Ar}$ & $\mathrm{P}$ & 12 & 0 & 12 & 12 & 66.8 & $8.7 \mathrm{~cm}$ & 5.8 & $25-35 / 5$ & $\begin{array}{l}2 \times \text { Fatigue, } \\
1 \times \text { bone pain }\end{array}$ & 0 & 89.8 & -2.8 & 100 \\
\hline Grubb (2018) [36] & $\mathrm{Ab}$ & $\mathrm{P}$ & 11 & 11 & 0 & 11 & 72 & $3.6 \mathrm{~cm}$ & 20.4 & $48-60 / 3$ & $1 \times$ Pyelo & 0 & NR & NR & 90.9 \\
\hline Kasuya (2018) [37] & $\mathrm{Ar}$ & $P$ & 19 & 15 & 4 & 19 & 67 & $3.6 \mathrm{~cm}$ & 79.2 & $\begin{array}{l}66-80 / 12-16 \\
\text { (CIRT) }\end{array}$ & 0 & $\begin{array}{l}1 \times \text { Dermatitis; } \\
2 \times \text { CKD }\end{array}$ & 42.7 & $-15.6^{\mathrm{b}}$ & 94.1 \\
\hline Kasuya (2019) [39] & $\mathrm{Ar}$ & $P$ & 8 & 7 & 1 & 8 & 71 & $4.3 \mathrm{~cm}$ & 43.1 & $\begin{array}{l}66-72 / 12 \\
\text { (CIRT) }\end{array}$ & 0 & 0 & 64.1 & -10.8 & 100 \\
\hline Funayama (2019) [38] & $\mathrm{Ar}$ & $P$ & 13 & 13 & 0 & 13 & 72 & $2.28 \mathrm{~cm}$ & 48.3 & $\begin{array}{l}60 \text { or } 70 / 10 \\
\text { (CIRT) }\end{array}$ & 0 & $2 \times \mathrm{CKD}$ & 51.9 & -16.8 & 92.3 \\
\hline
\end{tabular}


Table 2 - Baseline characteristics and outcomes: summary statistics.

\begin{tabular}{|c|c|c|c|c|c|}
\hline \multirow[t]{2}{*}{ Parameter } & \multirow[t]{2}{*}{ Reporting studies } & \multicolumn{2}{|c|}{$N$} & \multirow[t]{2}{*}{ Value } & \multirow[t]{2}{*}{ Measure of dispersion } \\
\hline & & Patients & Tumors & & \\
\hline Totals & 26 & 372 & 383 & - & - \\
\hline WeightedMeans & & & & Mean & Range \\
\hline Median follow-up (mo) & 25 & 358 & & 28 & $5.8-79.2$ \\
\hline Age (yr) & 19 & 303 & & 70.4 & $62-83$ \\
\hline Pre-SABR eGFR (ml/min) & 11 & 161 & & 59.0 & $28.7-89.8$ \\
\hline Tumor size $(\mathrm{cm})$ & 16 & & 202 & 4.6 & $2.3-9.5$ \\
\hline Weighted Percentages & & & & n (\%) & \\
\hline Stage & 26 & 372 & & & \\
\hline I-II & & & & $300(80)$ & - \\
\hline III-IV & & & & $72(20)$ & - \\
\hline RCC histology & 16 & & 242 & 191 (78.9) & - \\
\hline Random-EffectEstimates & & & & \%orMean & 95\% CI \\
\hline Local control & 25 & & 369 & $97.2 \%$ & $93.9-99.5 \%$ \\
\hline Toxicity (CTCAE grade $3-4$ ) & 23 & 287 & & $1.5 \%$ & $0.0-4.3 \%$ \\
\hline Post-SABR eGFR change & 8 & 56 & & $-7.7 \mathrm{ml} / \mathrm{min}$ & -12.5 to -2.8 \\
\hline
\end{tabular}

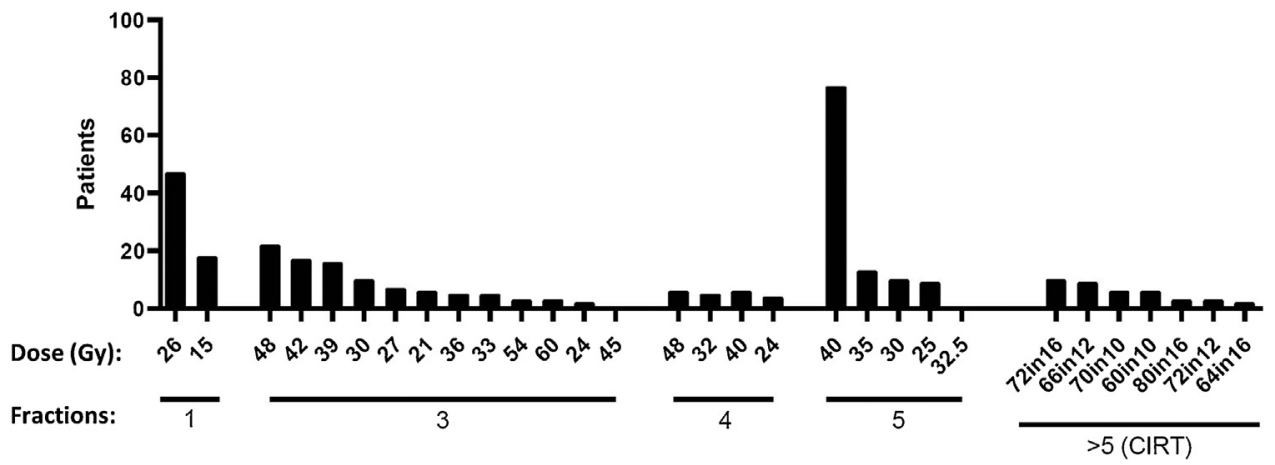

Fig. 3 - Dose-fractionation regimens. In studies reporting patient-specific dose and fractionation (22 studies, 333 patients), single-fraction ( $n=65$, three-fraction $(n=98)$, four-fraction $(n=21)$, and five-fraction $(n=110)$ regimens were utilized. Carbon ion radiotherapy $(C I R T)$ utilized regimens with more than five fractions $(n=39)$.

$I^{2}=20 \%$ and $Q$ test $p=0.19$, as shown in Figure 4A. LC rates ranged from $70 \%$ to $100 \%$ in the eligible studies. Local failures tended to occur in low-dose arms or where dose was compromised to mitigate toxicity [16,18,24,30]. Such dose dependency is commonly observed for SABR in other disease sites. It is also illustrated in RCC by the findings of Wang et al [41] demonstrating LC rates as low as $43.5 \%$ at $5 \mathrm{yr}$ when using fractional doses of 3-5 Gy (total: 36-51 Gy) for mostly large or locally invasive T3/4 RCC.

The LC rate demonstrated here for primary RCC SABR compares favorably with TA with consideration of tumor size and follow-up duration. A meta-analysis of 1375 lesions treated with RFA and CA reported LC rates of $87.1 \%$ and $94.8 \%$, respectively, with 18.7 mo of follow-up [40]. Therefore, potential concerns around follow-up length for SABR should take into consideration the longer follow-up observed in our meta-analysis, in which follow-up after SABR was 28.0 mo by comparison. Additionally, the mean tumor size for RFA/CA was $2.64 \mathrm{~cm}$ [40] compared with $4.6 \mathrm{~cm}$ for SABR in our meta-analysis. It is known that for TA modalities, T1b ( $>4 \mathrm{~cm}$ ) tumors are associated with higher local recurrence rates: up to $14.3 \%$ for RFA and $23 \%$ for CA
$[7,8]$. While not a direct comparison with these ablative modalities, our results at least demonstrate that SABR can achieve LC within a similar range-even with larger tumors and longer follow-up-thus justifying further comparative effectiveness research.

\subsection{Toxicity}

The random-effect estimate for CTCAE grade 3-4 toxicity was $1.5 \%$ (95\% CI: $0.0-4.3 \%)$; there was no heterogeneity present with $I^{2}=0 \%$ and $Q$ test $p=0.61$, as shown in Figure 4B. Treatment was generally well tolerated in all 23 studies reporting on toxicity. Out of 287 patients undergoing renal SABR, the majority of toxicity was mild nausea, fatigue, or dermatitis observed in $37.5 \%$ (grade 1 ) and $8.8 \%$ (grade 2) of patients (weighted percentages). Eight grade 3 events were reported, including possibly treatmentrelated pyelonephritis $(n=1)$ and gastric ulcer $(n=1)$. There were two grade 4 events $(0.9 \%)$ : duodenal ulcer $(n=1)$ and dermatitis $(n=1)$. Grade $3-4$ toxicity rates ranged from $0 \%$ to $25 \%$ in the eligible studies. There was no treatmentrelated mortality. 
A

Study

Correa, 2016

Hanzly, 2014

Svedman, 2006

McBride, 2013

Svedman, 2008

Wurzer, 2012

Wersall, 2005

Bietler, 2004

Grubb, 2018

Funayama, 2019

Sun, 2016

Qian, 2003

Gilson, 2006

Kasuya, 2018

Teh, 2007

Chebotareva, 2013

Nair, 2013

Lo, 2014

Staehler, 2015

Ponsky, 2015

Chang, 2016

Kaider-Person, 2017

Siva, 2017

Correa, 2018

Kasuya, 2019

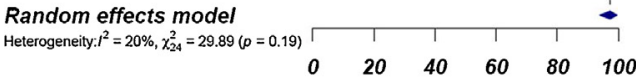

B

B

Local Control (\%)

Study

Wersall, 2005

Svedman, 2006

Teh, 2007

Svedman, 2008

Wurzer, 2012

Chebotareva, 2013

Nair, 2013

McBride, 2013

Lo, 2014

Hanzly, 2014

Staehler, 2015

Kaider-Person, 2017

Kasuya, 2019

Bietler, 2004

Siva, 2017

Kasuya, 2018

Singh, 2017

Correa, 2016

Grubb, 2018

Chang, 2016

Funayama, 2019

Ponsky, 2015

Correa, 2018

Random effects mode Heterogeneity: $I^{2}=0 \%, \chi_{22}^{2}=19.60(p=0.61)$

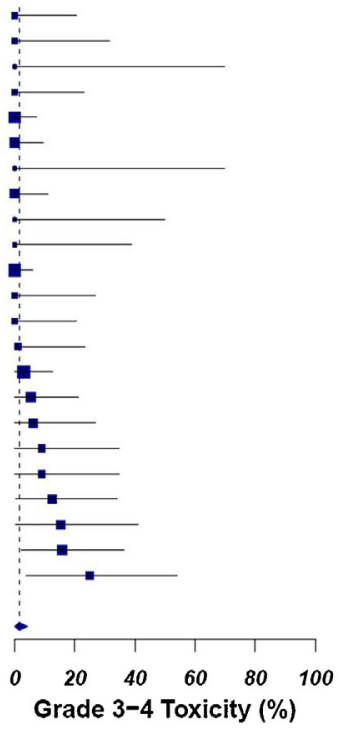

C
Local Control (\%) $\quad 95 \%$ C.I.

$\begin{array}{ll}70.0 & {[39.2 ; 94.1]} \\ 75.0 & {[20.7 ; 100.0]} \\ 79.0 & {[31.4 ; 100.0]} \\ 80.0 & {[55.4 ; 97.0]} \\ 85.7 & {[48.3 ; 100.0]} \\ 87.0 & {[69.6 ; 98.2]} \\ 87.5 & {[53.8 ; 100.0]} \\ 89.0 & {[58.4 ; 100.0]} \\ 90.9 & {[65.0 ; 100.0]} \\ 92.3 & {[69.9 ; 100.0]} \\ 92.7 & {[80.5 ; 99.6]} \\ 93.0 & {[76.6 ; 100.0]} \\ 94.0 & {[82.7 ; 99.9]} \\ 94.1 & {[77.7 ; 100.0]} \\ 100.0 & {[30.3 ; 100.0]} \\ 100.0 & {[90.7 ; 100.0]} \\ 100.0 & {[30.3 ; 100.0]} \\ 100.0 & {[50.0100 .0]} \\ 100.0 & {[94.2 ; 100.0]} \\ 100.0 & {[91.1 ; 100.0]} \\ 100.0 & {[89.5 ; 100.0]} \\ 100.0 & {[73.2 ; 100.0]} \\ 100.0 & {[94.9 ; 100.0]} \\ 100.0 & {[68.1 ; 100.0]} \\ 100.0 & {[79.6 ; 100.0]} \\ 97.2 & {[93.9 ; 99.5]}\end{array}$

Grade 3-4 Toxicity (\%) $95 \%$ C.I.

$\begin{array}{ll}0.0 & {[0.0 ; 20.4]} \\ 0.0 & {[0.0 ; 31.7]} \\ 0.0 & {[0.0 ; 69.7]} \\ 0.0 & {[0.0 ; 23.2]} \\ 0.0 & {[0.0 ; 7.3]} \\ 0.0 & {[0.0 ; 9.3]} \\ 0.0 & {[0.0 ; 69.7]} \\ 0.0 & {[0.0 ; 11.2]} \\ 0.0 & {[0.0 ; 50.0]} \\ 0.0 & {[0.0 ; 38.9]} \\ 0.0 & {[0.0 ; 5.8]} \\ 0.0 & {[0.0 ; 26.8]} \\ 0.0 & {[0.0 ; 20.4]} \\ 1.0 & {[0.0 ; 23.4]} \\ 3.0 & {[0.0 ; 12.5]} \\ 5.3 & {[0.0 ; 21.3]} \\ 6.2 & {[0.0 ; 26.7]} \\ 9.0 & {[0.0 ; 34.9]} \\ 9.0 & {[0.0 ; 34.9]} \\ 12.5 & {[0.3 ; 34.1]} \\ 15.4 & {[0.4 ; 41.0]} \\ 15.8 & {[2.3 ; 36.2]} \\ 25.0 & {[3.9 ; 53.9]} \\ 1.5 & {[0.0 ; 4.3]}\end{array}$

Study

Post-SABR Pre-SABR

Funayama et al., 2019

Kasuya et al., 2018

Kasuya et al., 2019

Siva et al., 2017

Lo et al., 2014

Correa et al., 2018

Correa et al., 2016

Nair et al., 2013

$\begin{array}{ll}35.2 & 51.9 \\ 27.1 & 42.7 \\ 51.9 & 64.1 \\ 43.2 & 54.7 \\ 22.0 & 28.7 \\ 87.0 & 89.8 \\ 47.1 & 48.6 \\ 44.0 & 38.0\end{array}$

Random effects model

Heterogeneity: $I^{2}=2 \%, \chi_{7}^{2}=7.15(p=0.41)$

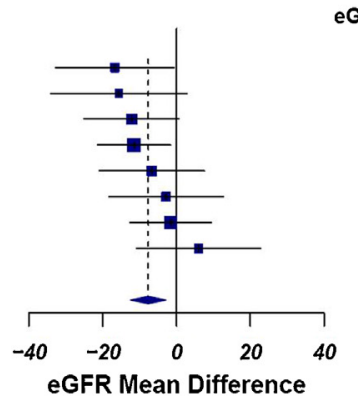

eGFR Mean Difference $\quad 95 \%$ C.I.

$\begin{array}{ll}-16.7 & {[-32.7 ;-0.7]} \\ -15.6 & {[-34.0 ; 2.8]} \\ -12.2 & {[-25.1 ; 0.7]} \\ -11.5 & {[-21.4 ;-1.6]} \\ -6.7 & {[-20.9 ; 7.5]} \\ -2.8 & {[-18.3 ; 12.7]} \\ -1.5 & {[-12.5 ; 9.5]} \\ 6.0 & {[-10.8 ; 22.8]} \\ -7.7 & {[-12.5 ;-2.8]}\end{array}$

eGFR Mean Difference

Fig. 4 - Primary and secondary outcomes. Forest plots depicting weighted random-effect estimates, $95 \%$ confidence intervals, and heterogeneity for (A) local control, (B) toxicity, and (C) renal function change after SABR. CI = confidence interval; eGFR = estimated glomerular filtration rate;

$\mathrm{SABR}=$ stereotactic ablative radiotherapy. 
The toxicity profile of SABR appears tolerable with very rare serious events, comparing favorably with thermal ablative treatments. Moreover, laparoscopic TA and percutaneous TA carry an added risk of periprocedural complications (approximately 10\%) such as hemorrhage, urine leak, or stricture [9]. Additionally, the risks of a general anesthetic or postprocedure hospitalization-which can be substantial in a medically frail, older population-are not applicable to SABR. Thus, from a patient's perspective, SABR may represent a potentially desirable, noninvasive option that appears well tolerated, particularly if additional prospective clinical trials replicate the low toxicity rates observed to date.

\subsection{Renal function}

The random-effect estimate for eGFR mean difference before and after SABR was $-7.7 \mathrm{ml} / \mathrm{min}(95 \% \mathrm{CI}:-12.5$ to $-2.8 \mathrm{ml} / \mathrm{min}$ ); there was no heterogeneity present with $I^{2}=2 \%$ and $Q$ test $p=0.41$, as shown in Figure 4C. Rates of eGFR change before and after SABR ranged from -16.7 to +6.0 . Most patients had some degree of renal function impairment before SABR (mild-moderate CKD), contributing to a weighted mean eGFR of $59.0 \mathrm{ml} / \mathrm{min}$ (range: 28.7$89.8 \mathrm{ml} / \mathrm{min}, n=161,11$ studies). Dialysis was required in $2.9 \%$ of patients (six of 206 patients in 17 studies reporting this), all of whom had pre-existing renal dysfunction (CKD stage 2-5). None of the 35 solitary kidney patients in this analysis required dialysis, suggesting that renal function may be adequately preserved even without compensation by the contralateral kidney. Of note, some included studies reported a late onset of renal dysfunction after SABR, with initial maintenance of baseline eGFR followed by a late decline [37,38].

Although direct comparison of renal function outcomes with surgical or TA management is difficult, our results are comparable with a previously reported large systematic review and meta-analysis comparing PN with TA, wherein median changes in eGFR (ranges) of $-6.2(-18$ to +4.1$)$ and $-4.9(-8.0$ to +1.5$) \mathrm{ml} / \mathrm{min}$, respectively, were demonstrated [42]. This was primarily based on the treatment of T1a tumors, whereas our meta-analysis comprised patients who were older with larger treated tumors, and with pre-existing renal dysfunction (average $59 \mathrm{ml} / \mathrm{min}$ ) and other comorbidities.

To maximally preserve renal function after SABR, it will be important to accurately estimate the magnitude of renal function decline-particularly in the ipsilateral, tumor-bearing kidney-as well as the kinetics of this decline. Some studies included in this review utilized split-function renal scans (eg, MAG3 or DMSA) for kidney-specific assessment and have demonstrated an expected but modest decrease in ipsilateral kidney filtration after SABR [30,33]. Siva et al [43] have also used ${ }^{51} \mathrm{Cr}$-EDTA clearance and ${ }^{99 \mathrm{~m}} \mathrm{Tc}$-DMSASPECT/CT spit-function nuclear medicine imaging to quantify GFR at the global, kidney-specific, and region-specific level. When SABR treatment plans were overlaid on functional imaging scans, regional nephropathy was clearly evident only in high-dose areas. Furthermore, the extent of GFR decline was inversely proportional to the conformality of SABR plans (ie, how rapidly the high-dose region "cools" beyond the target boundaries). Siva et al [43] also observed expected compensation by the contralateral (untreated) kidney after SABR, but bilateral decline in GFR, the latter secondary to global worsening of CKD in patients with pre-existing nephropathy. Overall, a dose-response relationship was established wherein every $10 \mathrm{~Gy}$ of physical dose to ipsilateral renal parenchyma predicts a GFR decrease of $25-39 \%$. Regarding timing, an initial plateau phase followed by late-onset renal dysfunction $\geq 1 \mathrm{yr}$ after SABR has been described previously $[43,44]$ and is also noted in studies included here [37,38]. Taken together, these data indicate that careful treatment planning to minimize high-dose exposure of ipsilateral kidney parenchyma plus long-term follow-up ideally using functional imaging (ie, MAG3 or DMSA) will be important to capture late renal function decline in the targeted kidney.

\subsection{Contemporary questions in primary RCC SABR}

\subsubsection{Post-treatment biopsy}

Although utilized in the TA literature, the role of post-SABR biopsy is unclear in primary RCC. In contrast to TA, SABR does not immediately obliterate tumor and vascular architecture but instead relies primarily upon DNA damage causing loss of proliferative capacity, with ongoing evolution of cell kill over a protracted time frame. Immediate histological changes (and loss of contrast enhancement) are therefore not seen after SABR. Correspondingly, available data suggest that biopsy after SABR can be misleading: in two studies where planned post-SABR biopsies were obtained at 6 and 9 mo, residual disease was present in most cases; however, no local failures were reported in the follow-up period [27,36]. For example, a patient with a positive biopsy at 11 mo after SABR did not undergo salvage therapy but experienced eventual tumor regression with a negative second biopsy [27]. In a separate study, a patient who underwent carbon ion SABR with stable disease noted at 29 mo died of unrelated causes and underwent autopsy, revealing only hemorrhagic necrosis throughout the remaining tumor mass [38]. Thus, early post-treatment biopsy may "overcall" the clinical significance of a positive result. These "viable" cells most likely represent residual, preapoptotic tumor cells that ultimately have lost proliferative potential and are nonclonogenic.

The discordance of post-RT histological assessment with clinical response is also seen in other disease sites. In rectal cancer, immediate versus delayed (4-8 wk) surgery after neoadjuvant RT ( $25 \mathrm{~Gy}$ in five fractions) correlated with $5 \times$ greater pathological complete response (pCR) rate $(11.8 \%$ vs 1.7\%) [45]. Likewise in anal cancer, pathological clearance after RT can take over 12 (up to 48) wk [46]. In early-stage lung cancer, where SABR is a definitive standard-of-care treatment for medically inoperable patients, LC rates approach 90\% [47] despite a discordant pCR rate of only $60 \%$ at early resection and pathological assessment (10 wk) [48]. In prostate cancer, an analogous situation with "SABRlike" brachytherapy exists wherein initially positive posttreatment biopsies do not correlate with local failure and 
ultimately convert to negative for malignancy at later time points $[49,50,51]$. Contemporary studies of SABR for prostate cancer also fit this paradigm, wherein maximal prostate-specific antigen (PSA) response can take 18-24 mo [52] and delayed post-treatment biopsy results (96\% negative at $36 \mathrm{mo}$ ) best correlates with high biochemical control rates (98\%) [53].

Therefore, the role, utility, and particularly timing of post-treatment biopsy are not well defined, particularly in the context of RCC. Although a very useful tool in assessing response following TA, biopsy does not appear to be well suited to post-SABR evaluation, given its much more gradual mechanism of cytotoxicity. While there may be a role for late biopsy, it may be obviated by imaging surveillance demonstrating an absence of radiographic progression over this time frame. Examples in the literature of postRT biopsy suggest that it is potentially misleading, prompting additional treatment and anxiety. Some patients with a positive biopsy after RCC SABR went on to receive salvage therapy, which may have been unnecessary $[27,36]$, possibly exposing them to undue risks and side effects. It is hoped that forthcoming data from clinical trials incorporating post-SABR biopsy will provide some clarity. At the present time, however, routine post-SABR biopsy should be avoided off-study, or used with caution and with recognition of the potential for overtreatment.

\subsubsection{Response assessment}

At the present time, $\mathrm{LC}$ is determined using $\mathrm{CT}$ imaging and size-based RECIST criteria, although this arguably has its limitations as an assessment tool. One concern is early "pseudoprogression" after SABR. A repeatedly observed phenomenon in RCC and other sites is an initial size increase that may be misinterpreted as disease progression, but may actually be due to treatment-induced inflammation $[23,27,29,32,38]$. This eventually plateaus (ie, stable disease) or gives way to tumor shrinkage. Regarding timing of response, SABR tends to cause slow decreases in size over months to years, the rate of which does not appear to depend on the dose [31,38]; consequently, the definition of LC in the setting must include stable disease. This is likely attributable to its mechanism of action involving slowly evolving tumor cell kill versus immediate physical destruction of tumor and vascular architecture. The latter is seen with TA and causes immediate changes in contrast enhancement pattern [9]. Such contrast enhancement losses are not seen with SABR, and thus ongoing enhancement-even increased enhancement in some cases-can be misinterpreted as persistent/recurrent disease although no correlation with local failure has been observed [31]. Thus, response assessment after SABR is challenging and demands close monitoring with frequent and long-term imaging follow-up.

What is desired are better imaging modalities or surrogate biomarkers to monitor response more accurately. Multiparametric MRI shows promise in this regard, as dynamic contrast enhancement findings at early time points correlate with long-term tumor shrinkage on conventional imaging [54]. Evidence also exists for the use of prostate-specific membrane antigen (PSMA)-based positron emission tomography (PET) scans (as well as traditional fluorodeoxyglucose [FDG] PET) in mRCC [55]. PSMA is highly expressed in RCC neovasculature, and thus PSMA-targeted radiotracers are effective at identifying lesions, often with greater avidity than FDG. Moreover, signal changes in metastases after SABR treatment correlates with response [55]. In the setting of primary RCC SABR, intrinsic expression of PSMA in normal renal parenchyma calls into question the utility of PSMA PET; however, it may still be suitable for post-SABR monitoring wherein signal absence could correlate with ablation of both tumor and in-field parenchyma. Thus, investigational imaging modalities may represent novel response assessment tools for RCC after SABR.

\subsubsection{Novel applications of renal SABR}

"Cytoreductive" SABR, or targeting the primary tumor in mRCC, is of interest in the era of contemporary immunotherapy. Although recent randomized trials in the targeted therapy era (CARMENA and SURTIME) raise questions regarding the use of cytoreductive nephrectomy $(\mathrm{CN})$, it remains an important component of management in some patients. For those who might benefit from $\mathrm{CN}$ but are not surgical candidates, SABR represents a possible alternative treatment that can be delivered safely and tolerably even to large or locally invasive primary tumors [30,35]. Moreover, cytoreductive SABR may additionally activate an antitumor immune response [34], leading to tumor neoantigen presentation and thus serving as an in situ vaccine to prime host immunity [10]. Given the increasing use of checkpoint inhibitor therapy in $\mathrm{MRCC}$, the combination of immunotherapy and SABR holds great potential to increase overall response rates, both locally and distantly via abscopal effects. Furthermore, the primary tumor is thought to harbor the greatest clonal heterogeneity in RCC-and by extension, the greatest diversity of neoantigens-theoretically making it a preferred target (vs smaller, more clonally homogeneous metastatic lesions). Thus, cytoreductive SABR is a nascent but promising concept worthy of further investigation in the era of immune checkpoint inhibition.

Another unique application of SABR in RCC is targeting inferior vena caval tumor thrombus (IVCTT). Hannan et al [56] have demonstrated this to be feasible and tolerable, with encouraging oncological results in a recent case series. Given the high-risk nature of thrombectomy surgery, SABR may thus be an option for patients with unresectable or recurrent IVCTT.

\subsection{Future research}

In light of the encouraging data for primary RCC SABR, additional prospective trials of primary RCC SABR have been launched. A ClinicalTrials.gov search revealed six ongoing phase II trials of primary RCC SABR at this time: NCT02853162, NCT03108703, NCT01890590, NCT02613819, NCT03747133, and NCT02782715 (a study of MRI-guided SABR plus microwave ablation of primary RCC). Sufficient equipoise for randomization has also led to the opening of a Canadian randomized controlled trial 
of SABR versus RFA (RADSTER, NCT03811665). There is also interest in comparative effectiveness research of SABR versus TA utilizing existing datasets, as randomized data are not immediately forthcoming.

With the maturity of data for safety and efficacy of SABR for primary RCC, its integration into multimodality management is also being investigated. One such safety lead-in phase II trial (NCT02473536) is evaluating the application of neoadjuvant SABR to IVCTT in locally advanced primary RCC prior to RN and thrombectomy; the rationale is eradication of live tumor emboli that may be shed during surgical resection, thus aiming to improve relapse-free survival.

\section{Conclusions}

SABR for primary RCC is supported by over two decades of prospective and retrospective data and an option worthy of consideration in certain patients. This population is older, with more comorbidities and at a higher risk of CKD progression. While these patients may be managed initially with AS, consideration of SABR is reasonable if delayed intervention is indicated. SABR may be the preferred option in centrally located near the renal pelvis or larger tumors that may be less suitable for TA. The evidence reviewed above suggests that even for such patients and tumors, SABR is highly effective, is well tolerated, and adequately preserves renal function. While direct comparison is not possible, SABR outcomes are at least within the range of existing treatment options. The convenience of a noninvasive therapy requiring few outpatient visits may also be attractive from a patient perspective. Whether the outcomes to date are reproducible in direct head-to-head comparisons with existing treatment options within prospective clinical trials remains to be seen. While challenges remain regarding the optimal response assessment tool after SABR, ongoing research into novel imaging modalities holds promise.

Author contributions: Shankar Siva and Rohann Correa had full access to all the data in the study and takes responsibility for the integrity of the data and the accuracy of the data analysis.

Study concept and design: Siva, Correa.

Acquisition of data: Correa, Louie, Siva.

Analysis and interpretation of data: Correa, Lehrer, Zaorsky, Siva, Louie.

Drafting of the manuscript: Correa, Siva.

Critical revision of the manuscript for important intellectual content: All authors.

Statistical analysis: Lehrer.

Obtaining funding: None.

Administrative, technical, or material support: Siva, Louie.

Supervision: Siva, Louie, Zaorsky.

Other: None.

Financial disclosures: Shankar Siva certifies that all conflicts of interest, including specific financial interests and relationships and affiliations relevant to the subject matter or materials discussed in the manuscript (eg, employment/affiliation, grants or funding, consultancies, honoraria, stock ownership or options, expert testimony, royalties, or patents filed, received, or pending), are the following: S. Siva has received a research grant from Varian Industries for kidney SABR, unrelated to the submitted work. A. Louie has received honoraria from Varian Medical Systems Inc. and AstraZeneca, unrelated to the submitted work. S. Lo is a member of the Elekta ICON Gamma Knife Expert Group, and has received research support from Elekta $\mathrm{AB}$ and travel expense reimbursement from Accuray Inc., unrelated to the submitted work.

Funding/Support and role of the sponsor: None.

\section{Appendix A. Supplementary data}

Supplementary material related to this article can be found, in the online version, at doi:https://doi.org/10. 1016/j.euf.2019.06.002.

\section{References}

[1] Capitanio U, Bensalah K, Bex A, et al. Epidemiology of renal cell carcinoma. Eur Urol 2019;75:74-84.

[2] Sun M, Abdollah F, Bianchi M, et al. A stage-for-stage and grade-forgrade analysis of cancer-specific mortality rates in renal cell carcinoma according to age: a competing-risks regression analysis. Eur Urol 2011;60:1152-9.

[3] Sun M, Becker A, Tian Z, et al. Management of localized kidney cancer: calculating cancer-specific mortality and competing risks of death for surgery and nonsurgical management. Eur Urol 2014;65:235-41.

[4] McIntosh AG, Ristau BT, Ruth K, et al. Active surveillance for localized renal masses: tumor growth, delayed intervention rates, and \&5-yr clinical outcomes. Eur Urol 2018;74:157-64.

[5] Bhindi B, Thompson RH, Lohse CM, et al. The probability of aggressive versus indolent histology based on renal tumor size: implications for surveillance and treatment. Eur Urol 2018;74:489-97.

[6] Ristau BT, Kutikov A, Uzzo RG, Smaldone MC. Active surveillance for small renal masses: when less is more. Eur Urol Focus 2016;2:660-8.

[7] Caputo PA, Zargar H, Ramirez D, et al. Cryoablation versus partial nephrectomy for clinical T1b renal tumors: a matched group comparative analysis. Eur Urol 2017;71:111-7.

[8] Psutka SP, Feldman AS, McDougal WS, McGovern FJ, Mueller P, Gervais DA. Long-term oncologic outcomes after radiofrequency ablation for T1 renal cell carcinoma. Eur Urol 2013;63:486-92.

[9] Kurup AN. Percutaneous ablation for small renal masses-complications. Semin Intervent Radiol 2014;31:42-9.

[10] Siva S, Kothari G, Muacevic A, et al. Radiotherapy for renal cell carcinoma: renaissance of an overlooked approach. Nat Rev Urol 2017;14:549-53.

[11] Walsh L, Stanfield JL, Cho LC, et al. Efficacy of ablative high-doseper-fraction radiation for implanted human renal cell cancer in a nude mouse model. Eur Urol 2006;50:795-800, discussion 800.

[12] Siva S, Pham D, Gill S, Corcoran NM, Foroudi F. A systematic review of stereotactic radiotherapy ablation for primary renal cell carcinoma. BJU Int 2012;110:E737-43.

[13] DerSimonian R, Laird N. Meta-analysis in clinical trials. Control Clin Trials 1986;7:177-88.

[14] Qian G, Lowry J, Silverman P, Grosman I, Makara D, Lederman G. Stereotactic extra-cranial radiosurgery for renal cell carcinoma. Int J Radiat Oncol Biol Phys 2003;57:S283.

[15] Beitler JJ, Makara D, Silverman P, Lederman G. Definitive, high-doseper-fraction, conformal, stereotactic external radiation for renal cell carcinoma. Am J Clin Oncol 2004;27:646-8.

[16] Wersall PJ, Blomgren H, Lax I, et al. Extracranial stereotactic radiotherapy for primary and metastatic renal cell carcinoma. Radiother Oncol 2005;77:88-95. 
[17] Gilson B, Lederman G, Qian G, Fastaia M, Cangiane L. 2249: Hypofractionated stereotactic extra-cranial radiosurgery (HFSR) for primary and metastatic renal cell carcinoma. Int J Radiat Oncol Biol Phys 2006;66:S349.

[18] Svedman C, Sandstrom P, Pisa P, et al. A prospective Phase II trial of using extracranial stereotactic radiotherapy in primary and metastatic renal cell carcinoma. Acta Oncol 2006;45:870-5.

[19] Teh B, Bloch C, Galli-Guevara M, et al. The treatment of primary and metastatic renal cell carcinoma (RCC) with image-guided stereotactic body radiation therapy (SBRT). Biomed Imaging Interv J 2007;3:e6.

[20] Svedman C, Karlsson K, Rutkowska E, et al. Stereotactic body radiotherapy of primary and metastatic renal lesions for patients with only one functioning kidney. Acta Oncol 2008;47:1578-83.

[21] Wurzer J, Kimmel B, Braga G, Pagnani A. Oc-0044 Efficacy of stereotactic body radiation therapy for primary renal cell carcinoma. Radiother Oncol 2012;103:S17.

[22] Chebotareva T, Spizhenko N, Buryk V, et al. PO-0730: Stereotactic ablative radiotherapy (SABR) of primary and metastatic renal lesions for patients with single kidney. Radiother Oncol 2013; 106:S279.

[23] Nair VJ, Szanto J, Vandervoort E, et al. CyberKnife for inoperable renal tumors: Canadian pioneering experience. Can J Urol 2013; 20:6944-9.

[24] McBride SM, Wagner AA, Kaplan ID. A phase 1 dose-escalation study of robotic radiosurgery in inoperable primary renal cell carcinoma. Int J Radiat Oncol Biol Phys 2013;87:S84.

[25] Lo CH, Huang WY, Chao HL, Lin KT, Jen YM. Novel application of stereotactic ablative radiotherapy using CyberKnife((R)) for earlystage renal cell carcinoma in patients with pre-existing chronic kidney disease: initial clinical experiences. Oncol Lett 2014;8: 355-60.

[26] Hanzly M, Creighton T, Mix M, et al. Stereotactic body radiotherapy for the treatment of renal tumors. Urol Case Rep 2014;2:147-9.

[27] Ponsky L, Lo SS, Zhang Y, et al. Phase I dose-escalation study of stereotactic body radiotherapy (SBRT) for poor surgical candidates with localized renal cell carcinoma. Radiother Oncol 2015;117: 183-7.

[28] Staehler M, Bader M, Schlenker B, et al. Single fraction radiosurgery for the treatment of renal tumors. J Urol 2015;193:771-5.

[29] Chang JH, Cheung P, Erler D, Sonier M, Korol R, Chu W. Stereotactic ablative body radiotherapy for primary renal cell carcinoma in nonsurgical candidates: initial clinical experience. Clin Oncol 2016;28: e109-14.

[30] Correa RJM, Rodrigues GB, Chen H, Warner A, Ahmad B, Louie AV. Stereotactic ablative radiotherapy (SABR) for large renal tumors: a retrospective case series evaluating clinical outcomes, toxicity, and technical considerations. Am J Clin Oncol 2018;41:568-75.

[31] Sun MR, Brook A, Powell MF, et al. Effect of stereotactic body radiotherapy on the growth kinetics and enhancement pattern of primary renal tumors. AJR Am J Roentgenol 2016;206:544-53.

[32] Kaidar-Person O, Price A, Schreiber E, Zagar TM, Chen RC. Stereotactic body radiotherapy for large primary renal cell carcinoma. Clin Genitourin Cancer 2017;15:e851-4.

[33] Siva S, Pham D, Kron T, et al. Stereotactic ablative body radiotherapy for inoperable primary kidney cancer: a prospective clinical trial. BJU Int 2017;120:623-30.

[34] Singh AK, Winslow TB, Kermany MH, et al. A pilot study of stereotactic body radiation therapy combined with cytoreductive nephrectomy for metastatic renal cell carcinoma. Clin Cancer Res 2017;23:5055-65.

[35] Correa RJM, Ahmad B, Warner A, et al. A prospective phase I doseescalation trial of stereotactic ablative radiotherapy (SABR) as an alternative to cytoreductive nephrectomy for inoperable patients with metastatic renal cell carcinoma. Radiat Oncol 2018;13:47.

[36] Grubb W, Ponsky L, Lo SS, et al. Dose escalation to 60 Gy in 3 fractions with stereotactic body radiation therapy (SBRT) for poor surgical candidates with localized renal cell carcinoma: expansion of a phase I study. Int J Radiat Oncol Biol Phys 2018;102:e91.

[37] Kasuya G, Tsuji H, Nomiya T, et al. Updated long-term outcomes after carbon-ion radiotherapy for primary renal cell carcinoma. Cancer Sci 2018;109:2873-80.

[38] Funayama S, Onishi H, Kuriyama $K$, et al. Renal cancer is not radioresistant: slowly but continuing shrinkage of the tumor after stereotactic body radiation therapy. Technol Cancer Res Treat 2019; 18:1533033818822329.

[39] Kasuya G, Tsuji H, Nomiya T, et al. Prospective clinical trial of 12fraction carbon-ion radiotherapy for primary renal cell carcinoma. Oncotarget 2019;10:76-81.

[40] Kunkle DA, Uzzo RG. Cryoablation or radiofrequency ablation of the small renal mass: a meta-analysis. Cancer 2008;113:2671-80.

[41] Wang YJ, Han TT, Xue JX, et al. Stereotactic gamma-ray body radiation therapy for asynchronous bilateral renal cell carcinoma. Radiol Med 2014;119:878-83.

[42] Patel HD, Pierorazio PM, Johnson MH, et al. Renal functional outcomes after surgery, ablation, and active surveillance of localized renal tumors: a systematic review and meta-analysis. Clin J Am Soc Nephrol 2017;12:1057-69.

[43] Siva S, Jackson P, Kron T, et al. Impact of stereotactic radiotherapy on kidney function in primary renal cell carcinoma: establishing a dose-response relationship. Radiother Oncol 2016;118:540-6.

[44] Jackson P, Foroudi F, Pham D, et al. Short communication: timeline of radiation-induced kidney function loss after stereotactic ablative body radiotherapy of renal cell carcinoma as evaluated by serial (99m)Tc-DMSA SPECT/CT. Radiat Oncol 2014;9:253.

[45] Pettersson D, Lorinc E, Holm T, et al. Tumour regression in the randomized Stockholm III Trial of radiotherapy regimens for rectal cancer. Br J Surg 2015;102:972-8, discussion 988.

[46] Musio D, De Felice F, Raffetto N, Tombolini V. Management of persistent anal canal carcinoma after combined-modality therapy: a clinical review. Radiat Oncol 2014;9:39.

[47] Timmerman R, Paulus R, Galvin J, et al. Stereotactic body radiation therapy for inoperable early stage lung cancer. JAMA 2010; 303:1070-6.

[48] Palma DA, Nguyen TK, Louie AV, et al. Measuring the integration of stereotactic ablative radiotherapy plus surgery for early-stage nonsmall cell lung cancer: a phase 2 clinical trial. JAMA Oncol 2019;5:681-8.

[49] Reed D, Wallner K, Merrick G, Buskirk S, True L. Clinical correlates to PSA spikes and positive repeat biopsies after prostate brachytherapy. Urology 2003;62:683-8.

[50] Prestidge BR, Hoak DC, Grimm PD, Ragde H, Cavanagh W, Blasko JC. Posttreatment biopsy results following interstitial brachytherapy in early-stage prostate cancer. Int J Radiat Oncol Biol Phys 1997;37: 31-9.

[51] Brawer MK. Radiation therapy failure in prostate cancer patients: risk factors and methods of detection. Rev Urol 2002;4(Suppl 2): S2-11.

[52] Fuller DB, Falchook AD, Crabtree T, et al. Phase 2 multicenter trial of heterogeneous-dosing stereotactic body radiotherapy for low- and intermediate-risk prostate cancer: 5-year outcomes. Eur Urol Oncol 2018;1:540-7.

[53] Loblaw A, Cheung P, D'Alimonte L, et al. Prostate stereotactic ablative body radiotherapy using a standard linear accelerator: toxicity, biochemical, and pathological outcomes. Radiother Oncol 2013;107:153-8. 
[54] Reynolds HM, Parameswaran BK, Finnegan ME, et al. Diffusion weighted and dynamic contrast enhanced MRI as an imaging biomarker for stereotactic ablative body radiotherapy (SABR) of primary renal cell carcinoma. PLoS One 2018;13:e0202387.

[55] Siva S, Callahan J, Pryor D, Martin J, Lawrentschuk N, Hofman MS. Utility of (68) Ga prostate specific membrane antigen-positron emission tomography in diagnosis and response assessment of recurrent renal cell carcinoma. J Med Imaging Radiat Oncol 2017;61:372-8.

[56] Hannan R, Margulis V, Chun SG, et al. Stereotactic radiation therapy of renal cancer inferior vena cava tumor thrombus. Cancer Biol Ther 2015;16:657-61. 


\section{University Library}

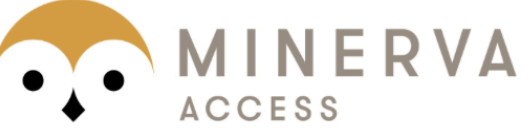

A gateway to Melbourne's research publications

Minerva Access is the Institutional Repository of The University of Melbourne

Author/s:

Correa, RJM;Louie, A;Zaorsky, NG;Lehrer, EJ;Ellis, R;Ponsky, L;Kaplan, I;Mahadevan, A;Chu, W;Swaminath, A;Hannan, R;Onishi, H;Teh, BS;Muacevic, A;Lo, SS;Staehler, M;Siva, S

Title:

The Emerging Role of Stereotactic Ablative Radiotherapy for Primary Renal Cell Carcinoma: A Systematic Review and Meta-Analysis

Date:

2019-11-01

Citation:

Correa, R. J. M., Louie, A., Zaorsky, N. G., Lehrer, E. J., Ellis, R., Ponsky, L., Kaplan, I., Mahadevan, A., Chu, W., Swaminath, A., Hannan, R., Onishi, H., Teh, B. S., Muacevic, A., Lo, S. S., Staehler, M. \& Siva, S. (2019). The Emerging Role of Stereotactic Ablative Radiotherapy for Primary Renal Cell Carcinoma: A Systematic Review and MetaAnalysis. EUROPEAN UROLOGY FOCUS, 5 (6), pp.958-969. https://doi.org/10.1016/ j.euf.2019.06.002.

Persistent Link:

http://hdl.handle.net/11343/280164

License:

CC BY 